\title{
A Comparative Electromyographic Analysis of Masseter and Temporalis Muscles in Edentulous Subjects
}

\author{
Krishna Prasad D. ${ }^{1}$, Annis Thomas ${ }^{2}$, Chethan Hegde ${ }^{3}$ \\ ${ }^{1}$ Department of Prosthodontics, Crown and Bridge, AB Shetty Memorial Institute of Dental Sciences, NITTE University, \\ Mangaluru, Karnataka, India. ${ }^{2}$ Department of Prosthodontics, Crown and Bridge, AB Shetty Memorial Institute of \\ Dental Sciences, NITTE University, Mangaluru, Karnataka, India. ${ }^{3}$ Department of Prosthodontics, Crown and Bridge, \\ AB Shetty Memorial Institute of Dental Sciences, NITTE University, Mangaluru, Karnataka, India.
}

\section{ABSTRACT}

\section{BACKGROUND}

All parts of skeletal system are held together and moved by skeletal muscles, which provide for locomotion for the individual to survive. Four pairs of muscles make a group of muscles of mastication: temporalis, masseter, medial and lateral pterygoid. Masseter is a rectangular muscle originating from the zygomatic arch extending downwards to lateral aspect of lower border of ramus. We wanted to evaluate the activity of masseter and temporalis muscle using electromyogram in subjects with balanced and non-balanced occlusion complete dentures.

\section{METHODS}

38 participants were selected and grouped into two as balanced and non-balanced occlusal denture subjects. Steps of fabricating a complete denture was followed along by addition of tracing in cases of balanced occlusion group. This was followed by recording the electromyographic readings at 1st appointment followed by an interval of 1 week and 3 weeks. These observations were then evaluated and compared for masseter and temporalis between the study groups.

\section{RESULTS}

The results show that there was an improvement in the muscle activity of temporalis and masseter when balanced group was observed following a period of 48 hours. However, the masseter showed noticeable improvement in non-balanced group at the end of 3 months when compared to balanced group.

\section{CONCLUSIONS}

This study was conducted under the hypothesis that there will be no change in the EMG activity of masseter and temporalis muscles in patients wearing complete dentures with and without balanced occlusion. Although, it was not statistically significant, the EMG recordings of masseter and temporalis, amongst balanced and non-balanced group, had a notable variance in the recordings to support that balanced occlusal scheme does not have a complete influence over all masticatory muscles.

\section{KEY WORDS}

Edentulous Patients, Electromyogram, Masseter and Temporalis
Corresponding Author:

Annis Thomas,

Postgraduate Student,

$A B$ Shetty Memorial Institute of Dental

Sciences, NITTE University, Mangaluru,

Karnataka, India.

E-mail: annisthomas@hotmail.com

DOI: 10.14260/jemds/2019/799

Financial or Other Competing Interests: None.

How to Cite This Article:

Prasad KD, Thomas A, Hegde C. A comparative electromyographic analysis of masseter and temporalis muscles in edentulous subjects. J. Evolution Med. Dent. Sci. 2019;8(49):3693-3697,

DOI: $10.14260 / \mathrm{jemds} / 2019 / 799$

Submission 24-07-2019,

Peer Review 24-09-2019,

Acceptance 01-10-2019,

Published 02-12-2019. 


\section{BACKGROUND}

All parts of skeletal system are held together and moved by skeletal muscles, which provide for locomotion for the individual to survive. Four pairs of muscles make a group of muscles of mastication: temporalis, masseter, medial and lateral pterygoid. Masseter is a rectangular muscle originating from zygomatic arch extending downwards to lateral aspect of lower border of ramus. It is a powerful muscle providing necessary force to chew efficiently. The superficial portion also aids in protrusion. Temporalis divides into 3 distinct areas according to fiber direction and ultimate function. Anterior fibers directed almost vertically when these fibers contract mandible is raised vertically, while middle fibers run obliquely across the lateral aspect of the skull and on contraction elevates and retrudes the mandible. Posterior fibers aligned almost horizontally, coming forward above the ear to join other temporalis fibers as they pass under the zygomatic arch. Because of the variant angulation of its muscle fibers, temporalis is capable of coordinating closing movements. It is thus a significant positioning muscle.(1)

Medial pterygoid on contraction, elevates the mandible and also aids in protrusion of mandible. However, unilateral contraction brings about mediotrusive movement of the mandible. Lateral pterygoid was described as having two distinct portions or bellies until studies proved otherwise. In this literature, lateral pterygoid is divided and is identified as two distinct and different muscles, namely inferiorly lateral pterygoid and superior lateral pterygoid. When the right and left pterygoids contact simultaneously condyles are pulled forward down the articular eminences and mandibular processes. Unilateral contraction brings about mediotrusive movement of the condyle and causes lateral movement of mandible to the opposite side. Along with mandibular depressors, the inferior lateral pterygoid lowers the mandible and the condyles glide forward and downward on the articular eminences. (2)

Balanced articulation is defined as the bilateral, simultaneous anterior and posterior occlusal contact of teeth in centric and eccentric positions. Balanced occlusion in complete denture is unique as it doesn't occur with natural teeth. The concept was originally put forth to enhance the retention of complete denture during mastication. Balance is now deemed necessary during many excursive movements such as swallowing saliva, closing to reseat dentures and bruxism performed by patients in between means. Hence, if the balance is not present, the bases could shift, tip or torque on their foundations during the eccentric movements and cause inflammation leading to accelerated bone resorption.(3) Though some authors, argue that these contacts other than mastication are not likely to be made with any great deal of force, it is seen that many patients enjoy comfort only when the eccentric balance is present. Complete edentulism not only hampers the mastication, aesthetics but also affects the psychological well-being of the patient.

Glossary of prosthodontics defines electromyography(4) as the graphic recording of electric potential of muscle. It can also be described as recording and study of fundamental electric properties of skeletal muscle using superficial or needle electrodes. In dentistry, uses of EMG is more common in TMJ disorder, dysfunction, dystonia, muscle disease of head and neck, cranial nerve lesion and seizure disorder ${ }^{5}$. Two methods of electromyography exist- surface and intramuscular. Surface electromyogram is used to assess muscle function by recording muscle activity from the surface over muscle in the skin using a pair of electrodes 6,7 . This type permits noninvasive investigation of bioelectrical phenomenon of muscular contraction.

\section{METHODS}

\section{Source of Data}

This in vivo study was conducted on a total of 38 patients using the following formula-

$$
n=\left[Z 1-\frac{\alpha}{2}+Z 1-\beta\right] x S \cdot D \cdot{ }^{2} /(\mu)^{2}
$$

From the age of 50 to 80 years. The muscles' activity was analysed using surface Electromyogram.

\section{Inclusion Criteria}

- Completely edentulous subjects.

- Subjects who are edentulous for at least 1 year.

- No Temporomandibular joint disorders.

- $\quad$ EMG was made 1-hour post consumption of food after 48 hours and in 1 week of insertion of complete dentures (immediate period) and after 3 months of insertion (delayed period).

\section{Exclusion Criteria}

- Patients with severely resorbed or flabby ridges.

- Subjects with uncontrolled systemic diseases.

- Patients with poor neuromuscular co-ordination.

Thirty-eight patients requiring complete dentures were selected and history was recorded to assess their medical history, dental history and habits to understand their pattern for loss of teeth. The normal procedure for fabricating a complete denture was followed including attachment of intra-oral tracers, Gothic-arch tracing and achieving interocclusal records. Patients were given post-insertion instructions and instructed to return for follow up after 48 hours. On completion of 48 hours, the patient was evaluated and enquired for problems with denture. After 1 hour of consumption of food, EMG recording was made to measure the activity of the muscles after denture insertion. After 3 months, the recordings were repeated for the same 1 hour after consumption of food.

\section{EMG Recording of Temporalis}

Two pairs of miniature-surface $\mathrm{Ag} / \mathrm{AgCl}$ electrodes were placed over the temporal muscle 1-inch posterior and 1-inch superior to outer canthus of the eye. The ground electrodes were placed over the skin of sternocleidomastoid. The recording was done at maximum intercuspal position.

\section{EMG Recording of Masseter}

Electrodes over the masseter muscle were placed $2 \mathrm{~cm}$ above the lower margin of mandible, halfway between the 
mandibular angle and anterior border of muscle was identified by palpation and the same procedure as mentioned above was followed to record EMG activity. The recordings are done at excursive movements apart from the maximum intercuspation.

\section{Statistical Analysis}

The data obtained was subjected to statistical analysis using IBM SPSS version 22. The mean and standard deviation of the muscles' tonicity was calculated, and these values were then compared using the 'Mann-Whitney $U$ ' test at a level of significance $(\mathrm{p})<0.05$.

\section{RESULTS}

Graph 1 shows the electromyographic recordings of Right temporalis muscle in balanced and non-balanced group. The observations are made at initiation of treatment and then during immediate period (48 hours, 1 week) and delayed period (3 months). Graph 2 shows the EMG recordings of Right temporalis during immediate and delayed periods of observation. Graph 3 shows observations of EMG of left temporalis in balanced and non-balanced groups made at initial appointment, immediate phase (48 hours, 1 week) and delayed period ( 3 months) of wearing dentures. Graph 4 depicts the EMG recordings of Left temporalis muscle made of subjects without dentures during follow up appointments of immediate and delayed phases.
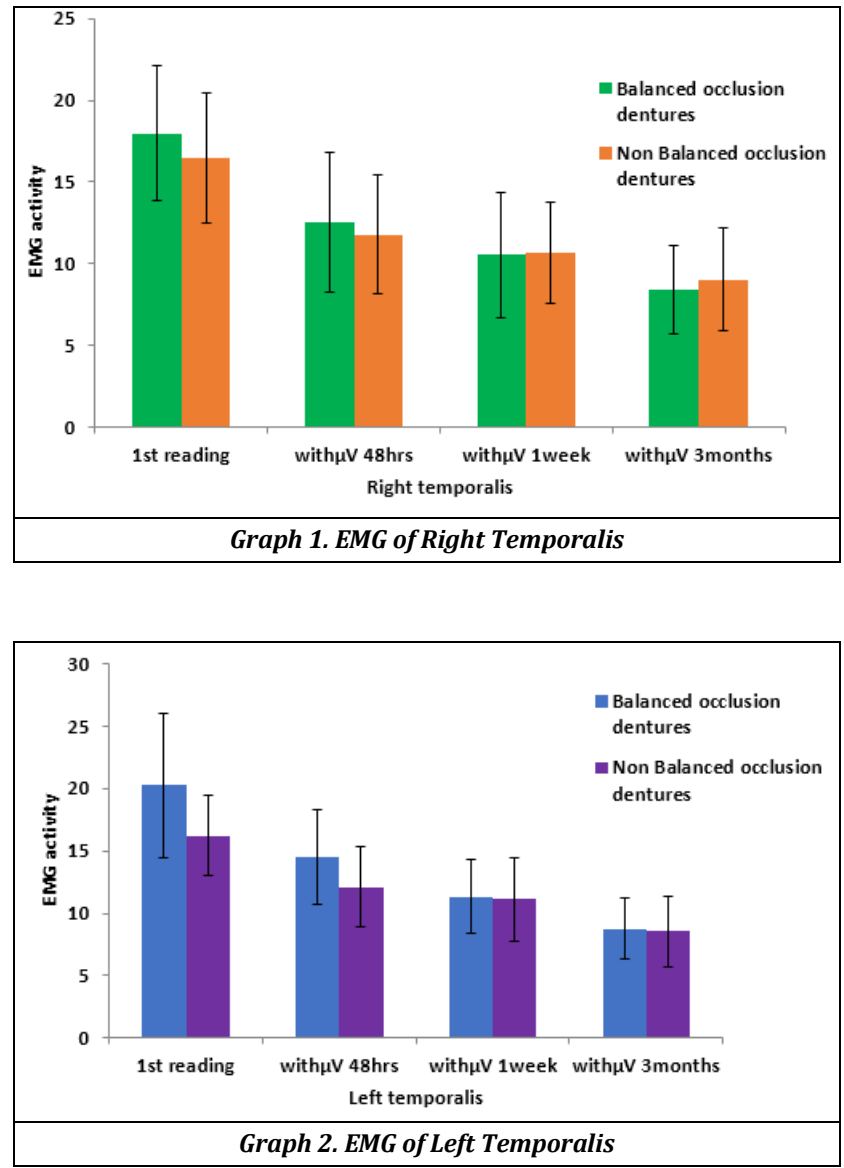
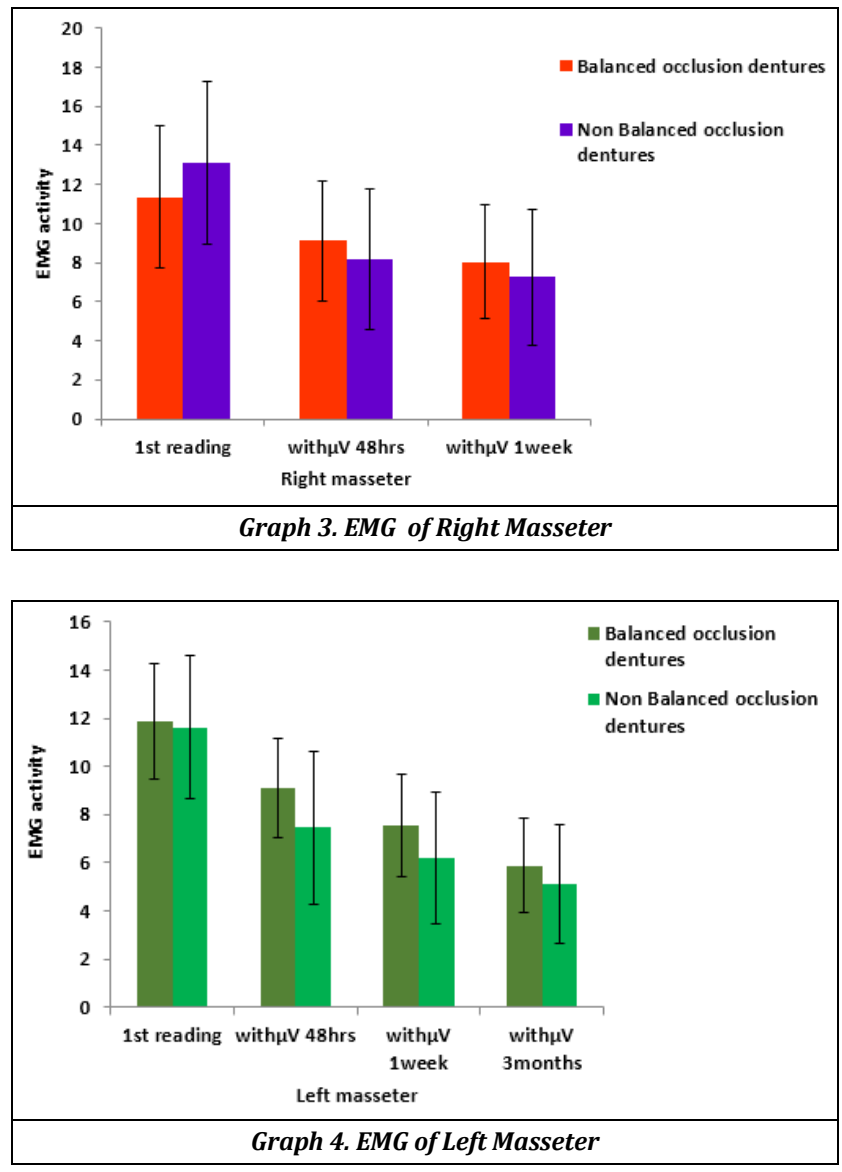

\section{DISCUSSION}

Mastication is highly coordinated neuromuscular process aided by dentition, skeletal components, temporomandibular joints, muscles and ligaments. Three components of skeletal system constitute the masticatory system- maxilla, mandible and temporal bone.(1) Temporalis muscle is a large muscle, its anterior one-third of this muscle upon contraction raise the mandible vertically, however, the middle one-third contract to elevate and retrude the mandible. In other words, because of variant angulation of its fibers, temporalis can coordinate the closing movements of the mandible. Masseter result in elevation of mandible into maximum intercuspation. It also provides necessary force for efficient chewing6,7.

In older patients, age results in reduced or almost impaired muscle force thereby reducing occlusal force, tactile sensations and chewing activity. Loss of periodontium brings about the change in neuromuscular aspect of mastication 8,9 . Human stomatognathic system brings adaptive changes when there is loss of teeth. The muscles change the masticatory pattern to adopt a comfortable chewing ability ${ }^{10}$. The required biting force also reduces as there is a change in dietary intake resulting in reduced muscle activity for chewing. To meet the remnant dentition or to chew in best comfortable position, position of mandible also changes ${ }^{11}$. One of the most common complaints presented by an edentulous patient is the inability to eat food as before. The masticatory efficiency is reduced by $80 \%$ in an edentulous person compared to dentulous person ${ }^{12}$.

Most dentate patient are present with either group function occlusion or mutually protected occlusion. However, 
neither can be incorporated into a complete denture prosthesis. Balanced occlusion is exclusive in complete denture where there is bilateral, simultaneous anterior and posterior occlusal contact in centric and eccentric position ${ }^{13}$. This was conceptualized to enhance stability of prosthesis during many excursive movements such as swallowing, closing to reseat the dentures and bruxism patients in exhibit between meals. However, studies conducted to compare the occlusal schemes contradict this notion. Many studies support canine guided occlusion for better retention of mandibular denture, aesthetic appearance and chewing ability.(14) Zhao Ketal(15) conducted a systematic review on clinical outcomes of various occlusal schemes for complete dentures. This review concluded that lingualized and canine guided occlusion can be successfully incorporated in complete denture fabrication. Lemos et al(16) in 2018, concluded his studied suggesting canine guided occlusion help to reduce muscle activity.

Many methods exist to assess the stomatognathic system, although to divulge into neurologic process of muscle activity, EMG has proved to be more useful ${ }^{17}$. Introduced by Moyers RE in 1949, electromyography is tactile tool which examines the electrical properties and determine the behaviour of masticatory muscle ${ }^{18}$. Miralles $\mathrm{R}$ et al(19) concluded in a study that unified EMG activity of both muscles during maximum voluntary clenching was notably reduced in patients with complete denture prosthesis than in natural dentates. Assessment of muscle function done by EMG is very accurate when compared to previous clinical observation. In the field of dentistry, applications of electromyography include as a diagnostic tool as well as a control signal for prosthetic device. Angelone et al.(20) in 1960, stated that EMG data is constitution of several variables. A F Roche implied that EMG findings indicate whether muscle is active, but one can assert some useful effect associated with muscle action by an unusual recording.

Surface EMG evaluates muscle function by recording muscle activity from over muscle surface. This is a noninvasive procedure. The surface EMG is presumed to give a quantitive and objective result. However, the surface EMG presents a few drawbacks- surface loosening of surface electrodes during nerve stimulation and errors caused by variation of positions of electrodes.

Many evidence-based controlled studies used extensive statistical analyses to confirm the safety, efficacy to evaluate resting and functional status of muscle and therefore is reliable and reproducible. Studies have been conducted which support surface EMG as tool for assessment of muscle function or disorders and the data obtained is reliable and reproducible. Clinical use of EMG has been studied over years in geriatrics. In 1960, Tallgren et al.(21) used EMG to study silent periods in edentulous subjects receiving immediate complete denture. Marina et al used EMG to study masticatory muscles after denture relining ${ }^{16}$. Surface EMG also been used in biofeedback concerning the awareness and control of nocturnal and diurnal parafunctional habits.

\section{Clinical Implications}

The occlusal schemes are important while constructing a complete denture. Based on the results drawn from this study, non-balanced occlusal scheme is better choice for improved muscle activity. It also enhances stability and retention of complete denture prosthesis.

\section{Limitations}

The principal limitation of the present study is that 3 months was not enough time to observe a significant change in muscle activity with the new complete dentures since, adaptation of neuromuscular system takes an extended time and may have an influential role on the EMG activity Indication of a dominant side in chewing pattern among the balanced group. Patients who have been edentulous for over 3-5 years and have adapted to a pattern required extra time to re-establish their musculature after changing the prosthesis. Precision of electromyographic data was less due to reduced exposure to the tools.

\section{CONCLUSIONS}

Balanced occlusal scheme does not have a uniform positive influence over the muscles of mastication in terms of muscle activity.

\section{ACKNOWLEDGEMENT}

The authors thank Prof. (Dr.) Suchetha P.S., Prof. (Dr.) Purosotham Chippala, Prof. (Dr.) Neevan D'souza (Associate Professor), Yenopoya Research Centre, Mangaluru.

\section{REFERENCES}

[1] Okeson JP. Fundamentals of occlusion and temporomandibular disorders. Mosby 1985.

[2] Rangarajan V, Gajapathi B, Yogesh PB, et al. Concepts of occlusion in prosthodontics: A literature review, part I. The Journal of Indian Prosthodontic Society 2015;15(3):200-5.

[3] Rangarajan V, Yogesh PB, Gajapathi B, et al. Concepts of occlusion in prosthodontics: a literature review, part II. The Journal of Indian Prosthodontic Society 2016;16(1):8-14.

[4] The glossary of prosthodontic terms: Ninth edition. The Journal of Prosthetic Dentistry 2017;117(Suppl 5):e1e105.

[5] Nishi SE, Basri R, Alam MK. Uses of electromyography in dentistry: an overview with meta-analysis. European Journal of Dentistry 2016;10(3):419-25.

[6] Roche AF. Functional anatomy of the muscles of mastication. The Journal of Prosthetic Dentistry 1963;13(3):548-70.

[7] Ashraf H. To determine the influence of the complete denture prosthesis on masticatory muscle activity in elderly patients: an in vivo study. International Journal of Prosthodontics and Restorative Dentistry 2011;1(1):3540.

[8] Alajbeg IZ, Valentic-Peruzovic M, Alajbeg I, et al. The influence of dental status on masticatory muscle activity in elderly patients. Int J Prosthodont 2005;18(4):333-8. 
[9] Grunert I, Kofler M, Gausch K, et al. Masseter and temporalis surface electromyography in patients wearing complete dentures comparing anterior and posterior occlusal concepts--a pilot study. J Oral Rehabil 1994;21(3):337-47.

[10] Sinnurkar S, Shakh SA, Nadiger R. Analysis of changes in bilateral masseter and anterior temporalis muscle efficiency in complete denture wearers - an EMG study. International Journal of Advanced Research 2017;5(2):2672-83.

[11] Piancino MG, Farina D, Talpone F, et al. Surface EMG of jaw-elevator muscles and chewing pattern in complete denture wearers. Journal of Oral Rehabilitation 2005;32(12):863-70.

[12] Gunne HS, Wall AK. The effect of new complete dentures on mastication and dietary intake. Acta Odontologica Scandinavica 1985;43(5):257-68.

[13] Maxwell D, Odang R, Koesmaningati H. Correlation of masticatory muscle activity with masticatory ability in complete denture patients with canine guidance and balanced occlusion. Journal of Physics: Conference Series. 2017;884:012015.

[14] Peroz I, Leuenberrg A, Haustein I, et al. Comparison between balanced occlusion and canine guidance in complete denure wearers - a clinical, randomized trial. Quintessence Int 2003;34(8):607-12.
[15] Zhao K, Mai QQ, Wang XD, et al. Occlusal designs on masticatory ability and patient satisfaction with complete denture: a systematic review. Journal of Dentistry 2013;41(11):1036-42.

[16] Lemos CAA, Verri FR, Gomes JML, et al. Bilateral balanced occlusion compared to other occlusal schemes in complete dentures: a systematic review. Journal of Oral Rehabilitation 2018;45(4):344-54.

[17] Baldisserotto SM, Da Trindade DCC, Loss JF, et al. Reliability of EMG activity in complete denture users during simulation of activities of daily living. Revista Odonto Ciência (Online) 2010;25(1):42-7.

[18] Goiato MC, Garcia AR, dos Santos DM. Electromyographic evaluation of masseter and anterior temporalis muscles in resting position and during maximum tooth clenching of edentulous patients before and after new complete dentures. Acta Odontol Latinoam 2007;20(2):67-72.

[19] Miralles R, Berger B, Ide W, et al. Comparative electromyographic study of elevator muscles in patients with complete dentures and natural dentition. J Oral Rehabil 1989;16(3):249-55.

[20] Angelone L, Clayton JA, Brandhorst WS. An approach to quantitative electromyography of the masseter muscle. Journal of Dental Research 1960;39(1):17-23.

[21] McCall W, Tallgren A, Ash MM Jr. EMG silent periods in immediate complete denture patients: a longitudinal study. Journal of Dental Research 1979;58(12):2353-9. 